\title{
Phytoprotection
}

\section{Voracité comparative de trois coccinelles prédatrices contre le tétranyque rouge du pommier [Acarina : Tetranychidae]}

\section{É. Lucas, S. Lapalme et D. Coderre}

Volume 78, numéro 3, 1997

URI : https://id.erudit.org/iderudit/706126ar

DOI : https://doi.org/10.7202/706126ar

Aller au sommaire du numéro

Éditeur(s)

Société de protection des plantes du Québec (SPPQ)l

ISSN

0031-9511 (imprimé)

1710-1603 (numérique)

Découvrir la revue

Citer cet article

Lucas, É., Lapalme, S. \& Coderre, D. (1997). Voracité comparative de trois coccinelles prédatrices contre le tétranyque rouge du pommier [Acarina : Tetranychidae]. Phytoprotection, 78(3), 117-123.

https://doi.org/10.7202/706126ar
Résumé de l'article

La voracité de la coccinelle à sept points, Coccinella septempunctata, de la coccinelle à quatorze points, Propylea quatuordecimpunctata, et de la coccinelle orientale, Harmonia axyridis, [Coleoptera : Coccinellidae] a été évaluée en laboratoire face au tétranyque rouge du pommier, Panonychus ulmi [Acarina : Tetranychidae]. Les trois espèces ont consommé le tétranyque rouge. $H$. axyridis était significativement plus vorace que les autres espèces et présente le potentiel le plus élevé comme ennemi naturel du tétranyque. Malgré sa grande taille, $C$. septempunctata possédait une voracité très faible, ce qui confirme son inefficacité comme agent de lutte face aux acariens phytophages. 


\title{
Voracité comparative de trois coccinelles prédatrices contre le tétranyque rouge du pommier [Acarina : Tetranychidae]
}

\author{
Éric Lucas, Sophie Lapalme et Daniel Coderre ${ }^{1}$
}

Reçu 1997-05-16; accepté 1998-01-14

PHYTOPROTECTION 78 : 117-123

La voracité de la coccinelle à sept points, Coccinella septempunctata, de la coccinelle à quatorze points, Propylea quatuordecimpunctata, et de la coccinelle orientale, Harmonia axyridis, [Coleoptera : Coccinellidae] a été évaluée en laboratoire face au tétranyque rouge du pommier, Panonychus ulmi [Acarina : Tetranychidae]. Les trois espèces ont consommé le tétranyque rouge. $H$. axyridis était significativement plus vorace que les autres espèces et présente le potentiel le plus élevé comme ennemi naturel du tétranyque. Malgré sa grande taille, C. septempunctata possédait une voracité très faible, ce qui confirme son inefficacité comme agent de lutte face aux acariens phytophages.

[Comparative voracity of three aphidophagous ladybeetles against the European red mite [Acarina : Tetranychidae]]

Voracity of the seven-spotted ladybeetle, Coccinella septempunctata, the fourteen-spotted ladybeetle, Propylea quatuordecimpunctata, and the oriental ladybeetle, Harmonia axyridis [Coleoptera : Coccinellidae], was evaluated in laboratory on the European red mite, Panonychus ulmi [Acarina : Tetranychidae]. The three species fed on the European red mite. $H$. axyridis was significantly more voracious than the other species and has the greater potential as a natural enemy of the mite. Despite its large size, C. septempunctata showed a very low voracity, confirming its inefficacy as a biological control agent against phytophagous mites.

\section{INTRODUCTION}

Sur 77 espèces de Coccinellidae présentes au Québec (Bousquet 1991), une douzaine se retrouvent en vergers de pommiers (Malus spp.) (Chouinard et al. 1992; Tourneur et al. 1992). Au sein de ce groupe, la coccinelle à sept points Coccinella septempunctata L., et la coccinelle à quatorze points, Propylea quatuordecimpunctata L. [Coleoptera : Coc- cinellidae] montrent une répartition générale dans la majorité des régions pomicoles du Québec (Chouinard et al. 1992). Les deux espèces sont principalement aphidiphages (lablokoff-Khnzorian 1982). C. septempunctata s'attaque aux cinq espèces de pucerons retrouvées en verger au Québec (Bonnemaison 1964; Bouchard et al. 1982; lablokoff-Khnzorian 1982; Lucas 1994; Olszack 1988), tandis que P. quatuorde-

1. Département des Sciences Biologiques, Université du Québec à Montréal, C.P. 8888, Succ. Centre-ville, Montréal (Québec), Canada H3C 3P8 
cimpunctata s'attaque au puceron vert du pommier, Aphis pomi de Geer, et au puceron des graminées Rhopalosiphum insertum (Sand.) [Homoptera : Aphididae] (lablokoff-Khnzorian 1982).

En 1994, Coderre et al. (1995) signalaient l'établissement d'une nouvelle coccinelle, Harmonia axyridis Pallas [Coleoptera: Coccinellidae], au Québec, en provenance des États-Unis. Cette espèce de grande taille, arboricole et polyphage, s'attaque à trois espèces de pucerons des vergers de pommiers : le puceron vert du pommier (A. pomi), le puceron de la spirée (Aphis citricola van der Goot) et le puceron lanigère du pommier (Eriosoma lanigerum Hausm.) [Homoptera : Aphididae] (Hodek 1973; Lucas 1994; Schanderl 1987). Les trois espèces de coccinelles, bien qu'exotiques (Chapin et Brou 1991; Dysart 1988; lablokoff-Khnzorian 1982), se sont bien adaptées dans le nord-est de l'Amérique du Nord et devraient cohabiter très bientôt dans les vergers de pommiers du Québec.

Les coccinelles aphidiphages, comme ces trois espèces, possèdent généralement une voracité élevée, tant au stade larvaire qu'au stade adulte. Récemment, Lucas et al. (1997b) ont évalué le potentiel de $C$. septempunctata et de $H$. axyridis face au tétranyque à deux points, Tetranychus urticae Koch [Acarina : Tetranychidae]. Bien que le tétranyque ait été attaqué même en présence de pucerons, les deux espèces prédatrices marquaient une préférence significative pour le puceron de la spirée, A. citricola (Lucas et al. 1997b). L'efficacité de prédation de la coccinelle à sept points diminuait avec l'augmentation de la proportion de tétranyques dans la diète disponible, illustrant sa mauvaise adaptation à ce nouveau type de proie, ce qui n'était pas le cas de l'espèce asiatique.

Ce travail vise à poursuivre la comparaison des coccinelles aphidiphages des vergers de pommiers, entre elles, et avec l'espèce $H$. axyridis, nouvellement introduite. Les trois espèces prédatrices ont été testées contre une proie de substitution, le tétranyque rouge du pommier, (Panonychus ulmi Koch) [Aca- rina : Tetranychidae]. Le tétranyque rouge est le principal acarien nuisible des vergers du Québec (Roy et Vincent 1992) et le tétranyque phytophage le plus commun dans les cultures pomicoles commerciales autant en Europe du Nord, qu'en Amérique du Sud ou au nord-est des États-Unis (Van de Vrie 1985).

\section{MATÉRIEL_ ET MÉTHODES}

Les adultes de $C$. septempunctata et de $P$. quatuordecimpunctata ont été récoltés dans un champ de maïs (Zea mays L.) de la région de Montréal (Québec, Canada) (lat. $45^{\circ} 30^{\prime} \mathrm{N}$, long. $73^{\circ} 36^{\prime} \mathrm{O}$ ) et les adultes de $H$. axyridis proviennent d'un élevage sur des oeufs d'Ephestia kuehniella Zeller [Lepidoptera : Pyralidae] (population d'origine chinoise). Les coccinelles étaient élevées sur le puceron de la spirée, A citricola $\left(25^{\circ} \mathrm{C}, 16 \mathrm{~h}\right.$ de lumière : $8 \mathrm{~h}$ d'obscurité, $\mathrm{HR}=70 \%$ ). Les tétranyques rouges provenaient de vergers de pommiers de la région de Saint-Hyacinthe (Québec, Canada) (lat. $45^{\circ} 39^{\prime} \mathrm{N}$, long. $72^{\circ} 56^{\prime} \mathrm{O}$ ).

Les pommiers (cv. Empire) étaient cultivés en serres. Au bout de 4 à 6 semaines, les racines et le feuillage étaient lavés puis séchés sous un jet d'air chaud. Les pommiers étaient taillés de façon à ne laisser que cinq feuilles de taille moyenne en parfait état, sans bourgeon, ni cotylédon. Le plant était fixé par la suite avec de la plasticine dans un orifice percé au fond d'un premier récipient de plastique de $8 \mathrm{~cm}$ de hauteur et de $15 \mathrm{~cm}$ de diam, lui-même placé dans un second de $15 \mathrm{~cm}$ de diam et de $15 \mathrm{~cm}$ de hauteur. Ce dernier contenait environ $1000 \mathrm{~cm}^{3}$ d'eau dans laquelle trempaient les racines du pommier. Le montage était fermé hermétiquement par un couvercle de plastique percé en son centre et recouvrarit un film plastique transparent et tendu.

Les coccinelles adultes étaient maintenues à jeun $24 \mathrm{~h}$ avant l'expérience. Soixante tétranyques rouges adultes étaient placés sur les feuilles du pommier préparé (répartis également sur toutes les feuilles). Après $15 \mathrm{~min}$, une 
coccinelle adulte était introduite dans le système. Les montages étaient placés à l'incubateur $\left(25^{\circ} \mathrm{C}, 16 \mathrm{~h}\right.$ de lumière : 8 h d'obscurité, $H R=70 \%)$. Après $24 \mathrm{~h}$, on effectuait un relevé à la loupe binoculaire des proies vivantes et des proies mortes non-consommées. Le nombre de proies manquantes indiquait la consommation de tétranyques par le prédateur.

Chacun des 21 réplicats contenait trois traitements: 1) une $H$. axyridis +60 tétranyques; 2 ) une $C$. septempunctata +60 tétranyques; 3 ) une $P$. quatuordecimpunctata +60 tétranyques; 4) un témoin (60 tétranyques, pas de prédateur). Les montages témoins évaluaient la mortalité naturelle. Les analyses ont été effectuées sur les données corrigées après ajustement par rapport aux témoins.

Les moyennes de proies consommées étaient comparées à l'aide d'une ANOVA à deux critères de classification (prédateur et sexe) (Scherrer 1984). La mortalité naturelle du témoin et des différents traitements (pour chaque prédateur) était également comparée avec une ANOVA à un critère (traitement). Les analyses étaient complétées par un test de comparaisons multiples de type Fisher non protégé (LSD). Les tests ont été réalisés à l'aide du logiciel SUPERANOVA (Abacus Concepts Inc. 1989).

\section{RÉSULTATS}

Les trois Coccinellidae consommaient P. ulmi (fig. 1). La voracité des trois espèces différait significativement (ANOVA, $F=64,92, \mathrm{dl}=2, p<0,0001$ ). $H$. axyridis a consommé en moyenne 48,91 tétranyques par $24 \mathrm{~h}$, soit six fois plus que $C$. septempunctata $(8,01)$ (LSD, $p=0,0001$ ), et significativement plus que $P$. quatuordecimpunctata $(34,16$ par 24 h) (LSD, $p=0,0001$ ). La consommation de $P$. quatuordecimpunctata dépassait significativement celle de $C$. septempunctata (LSD, $p=0,0001$ ). La voracité des mâles et des femelles était similaire (ANOVA, $F=0,025, \mathrm{dl}=1$, $p=0,8743$ ).

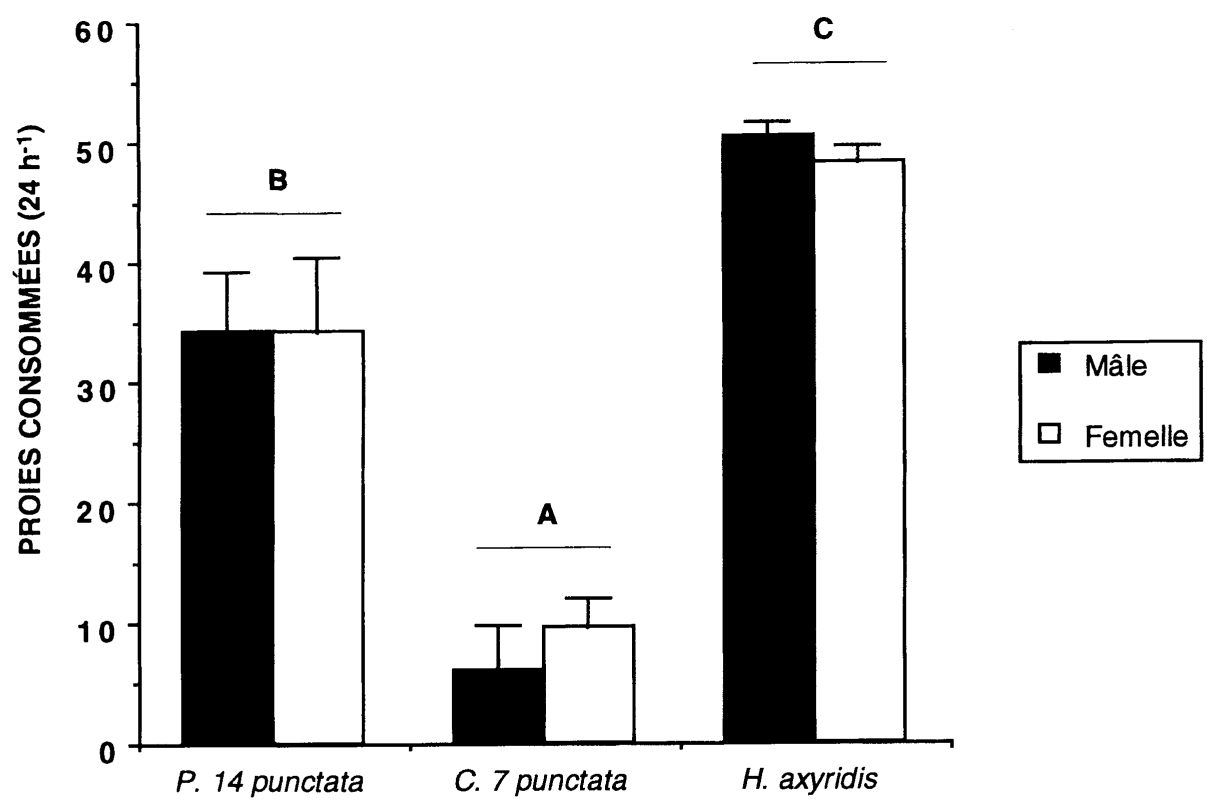

Figure 1. Voracité d'un adulte de Propylea quatuordecimpunctata (14 punctata), de Coccinella septumpunctata (7 punctata) et de Harmonia axyridis sur le Panonychus ulmi, en $24 \mathrm{~h}$. Abréviations: Des lettres différentes indiquent une différence significative entre les traitements $(\alpha=0,05)$. 


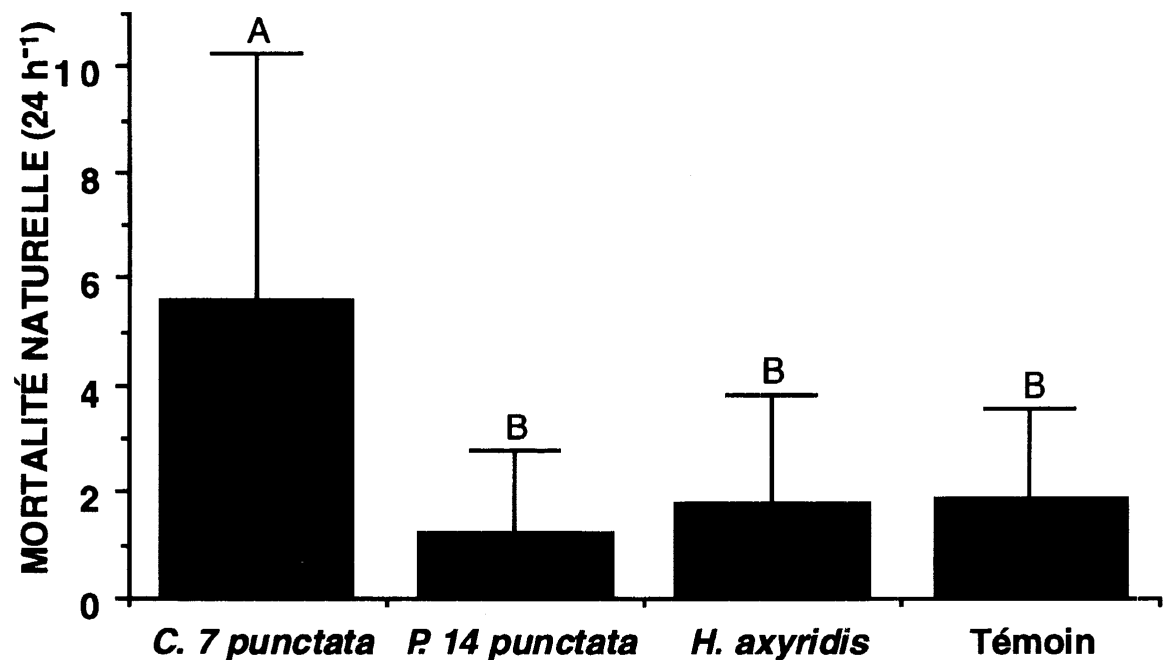

Figure 2. Nombre de Panonychus ulmi morts mais non-consommés, en présence d'un adulte de Coccinella septumpunctata ( 7 punctata), de Propylea quatuordecimpunctata (14 punctata), ou de Harmonia axyridis, ou en absence de prédateur sur plant de pommier en $24 \mathrm{~h}$. Abréviations: Des lettres différentes indiquent une différence significative entre les traitements $(\alpha=0,05)$.

La mortalité naturelle des tétranyques était similaire pour le témoin, $H$. axyridis et $P$. quatuordecimpunctata. Elle était cependant significativement supérieure en présence de $C$. septempunctata (ANOVA, $F=9.73, \mathrm{dl}=3, p<0.0001$ ) (fig. 2).

\section{DISCUSSION}

Pour un prédateur donné, il existe une taille minimale et maximale de proie acceptable (Sabelis 1992). Les prédateurs, particulièrement ceux effectuant de la digestion extra-orale (exemple: Reduviidae, Chrysopidae), profitent plus de l'utilisation de proies de grande taille (Cohen et Tang 1997). Les coccinelles aphidiphages constituent possiblement une exception à cette règle, puisqu'elles sont spécialisées dans les proies de très petite taille (e.g., pucerons) et qu'elles n'effectuent pas de digestion extraorale (Coderre et al., données nonpubliées). Les coccinelles $H$. axyridis $(4,80-7,50 \mathrm{~mm}), P$. quatuordecimpunctata $(3,50-5,20 \mathrm{~mm})$ (Gordon et Vandenberg 1991), C. septempunctata $(6,22$ $7,65 \mathrm{~mm}$ ) (Obrycki et Orr 1990) ont en effet consommé $P$. ulmi qui possède une taille inférieure à $10 \%$ de leur propre taille (adultes: $0,3 \mathrm{~mm}$ ) (Ministère de I'Agriculture du Canada 1977).

En absence de composés répulsifs ou toxiques séquestrés ou produits par les proies potentielles (Blum 1981), deux facteurs principaux semblent déterminer la voracité du prédateur, soit la spécificité alimentaire et la taille relative prédateur-proie. Les coccinelles aphidiphages sont généralement polyphages, mais leur spécificité varie seIon le type de proie (Hodek 1967; Majerus 1994). Elles sont spécialisées pour les proies optimales et polyphages pour les proies de substitution, c'est-à-dire ne permettant pas la maturation ovarienne (Hodek 1967; 1973). P. quatuordecimpunctata et $C$. septempunctata sont considérées essentiellement aphidiphages (Hodek 1973; lablokoff-Khnzorian 1982), tandis que $H$. axyridis serait plus polyphage (lablokoff-Khnzorian 1982). Selon nos résultats, le critère principal déterminant la voracité des coccinelles serait la spécificité alimentaire, ce qui expliquerait que, bien que de taille relativement similaire, $H_{\text {. }} a x y$ ridis soit plus vorace que $C$. septempunctata. Lors de la prédation du puceron du pois (Acyrthosiphon pisum 
(Harr.)), du puceron rose du pommier (Dysaphis plantaginea (Pass.)) [Homoptera : Aphididae] et d'A. pomi, la voracité des adultes de $C$. septempunctata était toujours supérieure à celle de $P$. quatuordecimpunctata, conformément à leur taille respective (Olszack 1988), ce qui n'est pas le cas ici. Pour expliquer la faible voracité de $C$. septempunctata, nous pouvons suspecter un refus de la proie par les adultes de la coccinelle à sept points. En effet, Putman (1957) a testé trois espèces du genre Coccinella et trouvé qu'aucun des 2,14 et 18 adultes testés ne dévorait de tétranyques rouges. Les consommations rapportées seraient dues possiblement à une période de "goûtage". Ajoutons que la mortalité naturelle de tétranyques était supérieure en présence de la coccinelle à sept points, ce qui pourrait s'expliquer par le fait que le faible nombre de proies consommées ne permettait pas la satiété, et qu'un prédateur affamé est généralement plus actif qu'un prédateur repu. Ce serait donc possiblement une plus grande activité de la coccinelle à sept points qui aurait provoqué la forte mortalité par des perturbations répétées provoquant la chute et la fuite des tétranyques. La sténophagie de $C$. septempunctata est confirmée par sa piètre performance sur le tétranyque à deux points (Lucas 1994; Lucas et al. 1997b). Pour P. quatuordecimpunctata, sa voracité supérieure nous indique qu'elle possède probablement un spectre alimentaire plus étendu que celui de $C$. septempunctata et que mentionné dans la littérature (lablokoff-Khnzorian 1982; mais voir Lucas 1994).

Le second facteur explicatif semble être la taille relative prédateur-proie. De manière implicite, plus la taille relative d'un prédateur par rapport à sa proie augmente, plus la biomasse consommée devrait augmenter. Ainsi chez les Coccinellidae, la voracité larvaire augmente avec la taille des larves (Hodek 1973). La voracité des coccinelles semble corrélée avec la taille relative pour $H$. axyridis et pour $P$. quatuordecimpunctata.

La voracité entre mâles et femelles n'était pas différente bien qu'habituel- lement les coccinelles aphidiphages femelles, particulièrement en période d'oviposition, se nourrissent plus que les mâles (Hodek 1973). Ceci pourrait s'expliquer par le fait que la proie est une proie de substitution et non une proie optimale, donc ne permettant pas la maturation ovarienne.

Du point de vue lutte biologique, cette étude ainsi que les travaux de Lucas et al. (1997b) montrent que C. septempunctata ne constitue pas un bon agent de lutte contre les tétranyques phytophages. $P$. quatuordecimpunctata, qui démontre une préférence pour la strate arbustive (0,5-2,0 m) (Hodek 1973) et possède une voracité limitée semble un moins bon candidat que $H$. axyridis plus vorace et arboricole. Néanmoins, étant donné les préférences alimentaires de la coccinelle orientale pour les pucerons (Lucas et al. 1997b), son efficacité devrait également être limitée. À titre comparatif, les coccinelles acariphages du genre Stethorus sont des prédateurs spécifiques des tétranyques (Putman et Herne 1966) et possèdent, malgré leur petite taille, des voracités comparables aux espèces étudiées ici (> 40 tétranyques adultes par jour pour un adulte) (McMurtry et al. 1970). Les coccinelles aphidiphages, bien que consommant les acariens (Collyer 1953; McMurtry et al. 1970; Putman 1957; Putman et Herne 1966), devraient avoir un rôle mineur dans leur contrôle, particulièrement en présence de pucerons. La seule espèce aphidiphage qui semble se développer normalement aux dépens des tétranyques est la coccinelle maculée, Coleomegilla maculata lengi (Timb.), mais qui est inféodée aux strates basses et donc peu utilisable en vergers de pommiers (Putman 1957). Malgré ces contraintes, les coccinelles étudiées ici peuvent présenter un potentiel pour la lutte contre les pucerons en vergers de pommiers (Olszak 1988) et la présence de tétranyques pourrait permettre la survie larvaire en absence d'autres proies potentielles (Collyer 1953).

Il existe également des dangers d'interférence ou de prédation intraguilde avec d'autres ennemis naturels de petite taille des tétranyques phytophages, 
tels que les phytoséiides ou les stades larvaires des coccinelles du genre Stethorus. Plusieurs études ont mis en évidence des cas de prédation intraguilde impliquant des agents de lutte biologique (Cloutier et Johnson 1993; Lucas et al. 1997a; Rosenheim et al. 1993).

\section{REMERCIEMENTS}

Cette étude a été réalisée grâce à une subvention du Ministère de l'Agriculture, des Pêcheries et de I'Alimentation du Québec (CORPAQ) à D. Coderre, à une bourse du Conseil de Recherche en Sciences Naturelles et Génie (CRSNG) du Canada, et une bourse de la Société d'entomologie du Canada octroyée à Éric Lucas. Sophie Lapalme a également bénéficié d'une bourse de recherche de premier cycle octroyée par le CRSNG.

\section{RÉFÉRENCES}

Abacus Concepts Inc. 1989. SuperAnova user's guide, BrainPower. Abacus Concepts Inc., Calabasas, Californie. 58 pp.

Blum, M.S. 1981. Chemical defenses of arthropods. Academic Press, New York. $562 \mathrm{pp}$.

Bonnemaison, L. 1964. Observations écologiques sur la coccinelle à 7 points (Coccinella septempunctata L.) dans la région parisienne [Col.]. Bull. Soc. Entomol. Fr. $69: 64-83$.

Bouchard, D., J.C. Tourneur et R.O. Paradis. 1982. Le complexe entomophage limitant les populations d'Aphis pomi De Geer (Homoptera : Aphididae) dans le sudouest du Québec. Données préliminaires. Ann. Soc. Entomol. Qué. 27 : 80-93.

Bousquet, Y. 1991. Checklist of beetles of Canada and Alaska. Agriculture Canada, Ottawa, Ontario. 430 pp.

Chapin, J.B. et V.A. Brou. 1991. Harmonia axyridis (Pallas), the third species of the genus to be found in the United States (Coleoptera : Coccinellidae). Proc. Entomol. Soc. Wash. $93: 630-635$.

Chouinard, G., M. Roy et C. Vincent. 1992. Ravageurs et faune auxiliaire des vergers de pommiers au Québec en 1992. Station de recherches, Agriculture Canada, Saint-Jean-sur-Richelieu, Québec. Résumé des recherches $21: 7-9$.
Cloutier, C. et S.G. Johnson. 1993. Predation by Orius tristicolor (Hemiptera : Anthocoridae) on Phytoseiulus persimilis (Acarina : Phytoseiidae): Testing for compatibility between biocontrol agents. Environ. Entomol. 22 : 477-482.

Coderre, D., E. Lucas et I. Gagné. 1995. The occurrence of Harmonia axyridis (Pallas) (Coleoptera : Coccinellidae) in Canada. Can. Entomol. 127 : 609-611.

Cohen, A.C. et R. Tang. 1997. Relative prey weight influences handling time arid biomass extraction in Sinea confusa and Zelus renardii (Heteroptera: Reduviidae). Environ. Entomol. 26 : 559-565.

Collyer, E. 1953. Biology of some predatory insects and mites with the fruit tree red spider (Metatetranychus ulmi (Koch)) in south-eastern England. IV. The predatormite relationship. J. Hortic. Sci. $28: 246-$ 259.

Dysart, R.J. 1988. The European lady beetle Propylea quatuordecimpunctata: New locality records for North America (Coleoptera : Coccinellidae). J. N.Y. Entomol. Soc. 96 : 119-121.

Gordon, R.D. et N. Vandenberg. 1991. Field guide to recently introduced species of Coccinellidae (Coleoptera) in North America, with a revised key to North American genera of Coccinellini. Proc. Entomol. Soc. Wash. $93: 845-864$.

Hodek, I. 1967. Food ecology of aphidophagous Coccinellidae. Entomophaga (Mémoire Hors Série) $3:$ 109-111.

Hodek, I. 1973. Biology of Coccinellidae. Publishing House, Prague, République tchèque. $260 \mathrm{pp}$.

lablokoff-Khnzorian, S.M. 1982. Les coccinelles; Coléoptères - Coccinellidae. Société nouvelle des éditions Boubée, Paris, France. 568 pp.

Lucas, E. 1994. Évaluation de l'efficacité de prédation des coccinelles Coccinella septempunctata L. et de Harmonia axyridis Pallas (Coleoptera: Coccinellidae) en tant qu'auxiliaires de lutte biologique en vergers de pommiers. Mémoire de maîtrise, Université du Québec à Montréal, Montréal (Québec). 106 pp.

Lucas, E., D. Coderre et J. Brodeur. 1997a. Instar-specific defense of Coleornegilla maculata lengi (Coleoptera : Coccinellidae): influence on attack success of the intraguild predator Chrysoperla rufilabris (Neuroptera: Chrysopidae). Entomophaga $42: 3-12$.

Lucas, E., D. Coderre et C. Vincent. 1997b. Voracity and feeding preferences of two aphidophagous coccinellids on Aphis citricola and Panonychus ulmi. Entomol. Exp. Appl. 85 : 151-159. 
Majerus, M.E.N. 1994. Ladybirds. HarperCollins Publishers, Toronto (Ontario). 367 pp.

McMurtry, J.A., C.B. Huffaker et M. van de Vrie. 1970. I. Tetranychid enemies: their biological characters and the impact of spray practices. Hilgardia $40: 331-373$.

Ministère de l'agriculture du Canada. 1977. Identification des insectes; feuillet $n^{\circ} 25$ : le tétranyque rouge du pommier, Panonychus ulmi (Koch). Ottawa, Ontario. $1 \mathrm{p}$.

Obrycki, J.J. et C.J. Orr. 1990. Suitability of three prey species for nearctic populations of Coccinella septempunctata, Hippodamia variegata, and Propylea quatuordecimpunctata (Coleoptera : Coccinellidae). J. Econ. Entomol. 83 : 1292-1297.

Olszack, R.W. 1988. Voracity and developement of three species of Coccinellidae preying upon different species of aphids. Pages 47-53 in E. Niemczyk et A.F.G. Dixon (réd.), Ecology and effectiveness of Aphidophaga. SPB Academic Publishing, The Hague.

Putman, W.L. 1957. Laboratory studies on the food of some Coccinellids (Coleoptera) found in Ontario Peach Orchards. Can. Entomol. 89 : 572-579.

Putman, W.L. et D.H.C. Herne. 1966. The role of predators and other biotic agents in regulating the population density of phytophagous mites in Ontario peach orchards. Can. Entomol. 98 : 808-820.

Rosenheim, J.A., L. R. Wilhoit et C.A. Armer. 1993. Influence of intraguild predation among generalist insect predators on the suppression of an herbivore population. Oecologia 96 : 439-449.
Roy, M. et C. Vincent. 1992. Les principaux acariens phytophages en pomiculture au Québec. Points de vue sur la protection des vergers au Québec. Agriculture $\mathrm{Ca}$ nada, Saint-Jean-sur-Richelieu (Québec). Bull. Technique 26 : 37-40.

Sabelis, M. W. 1992. Predatory Arthropods. Pages 225-264 in M.J. Crawley (réd.) Natural enemies. Blackwell Scientific Publ., Boston, Massachusetts.

Schanderl, H. 1987. Détermination des conditions optimales d'élevage de la coccinelle Harmonia axyridis Pallas (Coleoptera : Coccinellidae) et possibilité d'une production continue à l'aide d'une proie de substitution, les oeufs d'Ephestia kuehniella Zeller (Lepidoptera: Pyralidae). Thèse doctorale de l'Université de droit, d'économie et des sciences d'AixMarseilles, Marseilles, France. 139 pp.

Scherrer, B. 1984. Biostatistique. Gaëtan Morin éditeur, Chicoutimi (Québec). 868 $\mathrm{pp}$.

Tourneur, J.C., D. Bouchard et J.G. Pilon. 1992. Le complexe des ennemis naturels des pucerons en pommeraie au Québec. Pages 179-193 in C. Vincent et D. Coderre (réd.), La lutte biologique. Gaëtan Morin éditeur, Boucherville (Québec).

Van de Vrie, M. 1985. Apple. Pages 311 325 in W. Helle et M.W. Sabelis (réd.), World crop pests: spider mites, their biology, natural enemies and control. Vol. 1B. Elsevier, New York. 